\title{
Trapped waves in thin elastic plates
}

\author{
R. Porter \\ School of Mathematics, University of Bristol, Bristol, BS8 1TW, UK.
}

\begin{abstract}
In this paper we present new results which illustrate the existence of trapped modes on a thin elastic plate, which is infinitely long, of finite uniform width and simply-supported along the two parallel edges. The plates contain a circular cut-out on the centreline of the strip whose edges are either free or clamped. The trapped waves describe time-harmonic vibrations of a frequency below a cut-off frequency which are localised to the circular cut-out and decay along the strip. Results show that whilst no trapped waves occur for a circular hole with a clamped edge, for a hole with a free edge trapped waves occur in all four possible modes of symmetry.
\end{abstract}

Key words: Thin elastic plates, trapped waves, circular holes, simply-supported strip.

\section{Introduction and background}

Trapped waves are a ubiquitous feature in all types of wave theories from gravity water waves, acoustics, electromagnetics, optics, quantum mechanics and elasticity.

The term 'trapped wave' is used broadly to describe any oscillation or vibration in a system which is localised in space by some feature (geometric or otherwise) of the governing system. The particular mechanism giving rise to a trapped wave, and the subsequent form the trapped wave takes can be very different depending upon the type of problem being considered. For example, in its basic form, Anderson localisation proves the existence of localised waves in a system of randomly-placed scatterers in a one-dimensional waveguide. Fabry-Perot resonantors exploit stop-bands in infinite periodic structures to trap waves between two semi-infinite periodic structures (see [1], for example). In water waves, 'edge waves' travel along the shoreline of a plane beach, but not away from it (see [2]; there are many other examples of trapping in water wave theory). Similarly, in elasticity, Rayleigh-waves are confined close to the surface of a semi-infinite elastic solid,

Email address: richard.porter@bris.ac.uk (R. Porter).

Preprint submitted to Elsevier

19 September 2006 
whilst edge waves have been shown to occur on thin semi-infinite elastic plates (see later discussion).

In some examples, the possibility of the existence of trapped waves can be motivated by some relatively straightforward arguments; there may be a 'cut-off' frequency below which waves cannot radiate to infinity (often associated with waveguides) or some deconstructive interference between scatterers in infinite arrays (as in Anderson localisation or Fabry-Perot resonators). In other examples trapped waves are much more difficult to argue. For example, in the two-dimensional water wave problem, [3] demonstrated a new type of trapped wave; previously much effort had been directed towards trying to prove the non-existence of such trapped waves.

In nearly all cases, the presence of a trapped wave can have significant implications. In certain circumstances, it is something that can be exploited (Fabry-Perot resonanters being a good example) and in other cases, it should be avoided (acoustic resonances in turbomachinery, for example).

In this paper, we focus upon trapped waves in elastic plates which are confined to lie within a strip of uniform width. We briefly draw the attention of the reader to the recent work of researchers such as [4], [5] and [6]. These authors (also see references therein) have considered trapped waves in elastic waveguides which are either bent or which vary topographically. This body of work uses the full equations of elasticity to formulate the problem and the trapping is in the form of two-dimensional compressional or shear waves within the waveguide, which is used as a representation of the cross-section of a thin plate. In contrast, in this paper we consider the out-of-plane vibrations of a thin elastic plate described by Kirchhoff theory, in which variations in physical quantities across the plate are averaged.

Using classical Kirchhoff theory (see [7]), the equation of motion of a thin elastic plate is described in terms of its displacement, $W(x, y, t)$, perpendicular to the $(x, y)$-plane it occupies in equilibrium is given by

$$
-D \Delta^{2} W=m W_{t t}
$$

where $\Delta=\partial_{x x}+\partial_{y y}$ is the two-dimensional Laplacian and $t$ is time. The flexural rigidity of the plate is $D=\frac{1}{12} E h^{3} /\left(1-\nu^{2}\right)$, and its mass per unit area is $m=\rho h$, where $E, \rho$, $h$ are Young's modulus, the plate density and plate thickness and $\nu$ is Poisson's ratio.

Assuming a time-harmonic variation of angular frequency $\omega$, and writing $W(x, y, t)=$ $\Re\left\{w(x, y) \mathrm{e}^{-\mathrm{i} \omega t}\right\}$ we have

$$
\left(\Delta^{2}-k^{4}\right) w=0
$$

where $k^{4}=m \omega^{2} / D$ and $k$ is the wavenumber of free waves on the elastic plate.

Trapped waves are known to exist along the edge of a thin semi-infinite elastic plate ([8] and [9]). It proves useful to briefly revisit this problem. The general solution, in terms of unknown constants $A$ and $B$, satisfying the plate equation (1) in $x>0$ is $w(x, y)=\mathrm{e}^{ \pm \mathrm{i} l y}\left(A \mathrm{e}^{-\left(l^{2}-k^{2}\right)^{1 / 2} x}+B \mathrm{e}^{-\left(l^{2}+k^{2}\right)^{1 / 2} x}\right)$ such that $w \rightarrow 0$ as $x \rightarrow \infty$ provided that the wavenumber $l$ is chosen to satisfy $l>k$. If the edge, $x=0$, is clamped, implying the application of the conditions $w(0, y)=w_{x}(0, y)=0$, then it immediately becomes clear that no non-trivial solutions exist. However, if the edge is free, the conditions to be applied on $x=0$ are $w_{x x}+\nu w_{y y}=0, w_{x x x}+(2-\nu) w_{x y y}=0$, and subsequent (more lengthy) calculations reveal that non-trivial solutions do exist and are defined by the dispersion relation 


$$
\left(\frac{l}{k}\right)^{4}=\frac{1-3 \nu+2 \sqrt{1-2 \nu+2 \nu^{2}}}{(1-\nu)^{2}(3+\nu)} .
$$

For example, with $\nu=\frac{1}{3}$ (a realistic value for many materials) $l=(81 / 80)^{1 / 8} k \approx$ $1.00155 k$. More generally, $(l / k)^{4} \sim 1+\frac{1}{16} \nu^{4}+O\left(\nu^{5}\right)$ follows from (2), and the value of $k$ is generally only just below that of $l$. By superimposing two waves with variation in $y$ of $\mathrm{e}^{ \pm \mathrm{i} l y}$, we get

$$
w(x, y)=A \sin (l y)\left(\mathrm{e}^{-\left(l^{2}-k^{2}\right)^{1 / 2} x}+\left(\frac{k^{2}-(1-\nu) l^{2}}{k^{2}+(1-\nu) l^{2}}\right) \mathrm{e}^{-\left(l^{2}+k^{2}\right)^{1 / 2} x}\right) .
$$

This is a trapped mode, in which the wave energy is localised along the edge $x=0$, and decays exponentially (albeit very slowly) away from it. Such trapped waves (in this context often referred to as edge waves) are important in scattering by cracks in plates, see [10], for example. They have also been shown to exist for more complex plate theories (see [11], [12], [13]) and for plates with fluid loading [14], [15].

By choosing $l=\pi / 2 d$ or $l=\pi / d$ in (3), the trapped wave is equivalent to the vibrations on a thin elastic plate confined within a semi-infinite waveguide of width $2 d$, and upon whose parallel sides $y=0, y=2 d$ the plate is freely supported (i.e. $w=w_{y y}=0$ ). For $l=\pi / 2 d$, the modes described by (3) are symmetric across the centre of the guide $(y=d)$ whilst, for $l=\pi / d$, the modes are antisymmetric across the guide. The corresponding values of $k d$ for trapped modes defined by (2) are less than $\frac{1}{2} \pi$ or $\pi$ for the cases of symmetric and antisymmetric modes, a point that is pertinent later.

This problem therefore represents the most trivial example of the existence of trapped modes in elastic plates in a uniform strip. The fact that a free edge at $x=0$ supports trapped waves whilst a clamped edge does not has relevance to the new results we present here.

Another possibility, considered recently by [16] is to constrain a point in the centre of the guide. [16] assumed the point is attached by a mass/spring system to a fixed mooring point. Within this model, the point can be pinned by letting the mass or spring constant tend to infinity. It was proved in [16] that trapped modes do not exist for a pinned point on the centreline of a waveguide, but a combination of finite non-zero mass and spring constants constraining the motion of a point on the centreline would give rise to trapped modes. This type of trapped mode in which the inhomogeneity giving rise to trapping has its own dynamic properties has a recent analogue in water waves, where [17] has called them motion trapping modes (buoyancy and hydrodynamic forces providing spring and added-mass terms), as opposed to the more traditional trapping modes in water waves, in the presence of a fixed structure, as exemplified by [3].

In this paper, we consider a strip of uniform width, $2 d$, freely-supported on its parallel sides and which extends to infinity in both directions. We consider the possibility of trapped modes when some alteration is made to the elastic plate within the guide. In the example of trapped waves along a semi-infinite plate described previously, this alteration is simply to divide the guide in two by a cut along $x=0$, perpendicular to the sides of the guide. In contrast, clamping along this line does not give trapped modes, nor does pinning a point at the centre of the guide.

We aim to broaden the range of examples in which trapped waves can be found, and this is done by considering removing a circle from the centre of the strip and considering the effect of either clamping the edge or having a free edge. Circular cut-outs or inclusions are common in engineering to assist in weight-saving or reinforcement. For example, [18] 


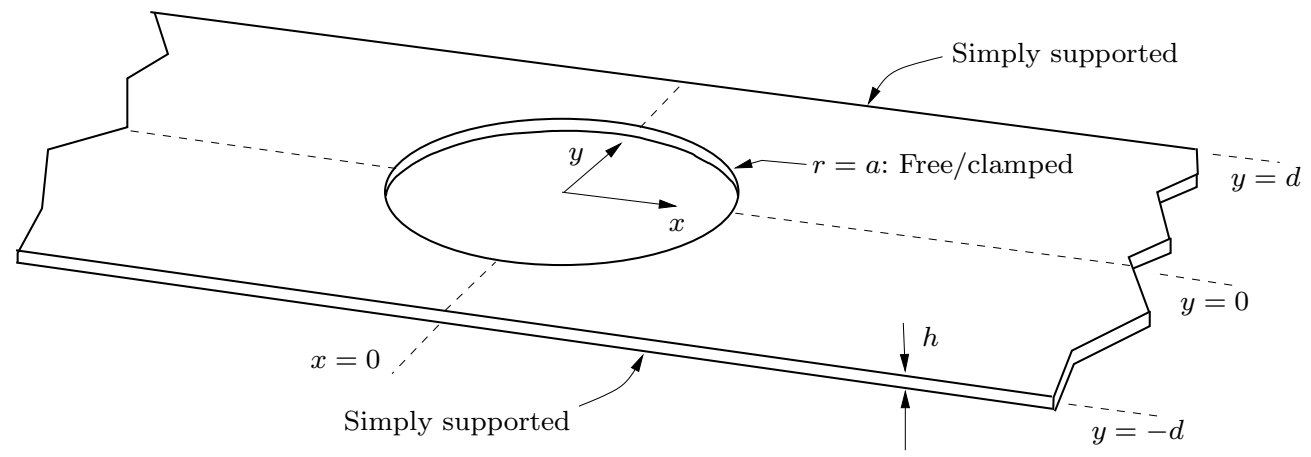

Fig. 1. Geometry of the problem.

consider plane wave scattering by circular cut-outs and inclusions in an infinite plate. Whilst the freely-supported conditions on the edges of the strip considered here are more artificial in terms of engineering applications than clamped or free edges, the principles used here allow us to speculate as to the existence of trapped modes in those cases. The method presented in the paper uses so-called multipole expansions (terminology derived from water wave theory) and follows most closely the work of [19] who investigated trapped modes due to vertical cylinders on the centreline of a channel.

\section{Formulation of the problem}

We consider an infinitely-long rectangular strip $-d<y<d,-\infty<x<\infty$ occupied by a thin elastic plate whose time-harmonic vibrations described by the function $w(x, y)$ are perpendicular to the plane it occupies in equilibrium. In the strip (1) is satisfied by $w(x, y)$.

On the lateral boundaries of the strip the elastic plate is simply (freely) supported, so that

$$
w=0, \quad \text { and } \quad \Delta w-\nu_{1} w_{x x} \equiv \Delta w=0, \quad \text { on }|y|=d,-\infty<x<\infty
$$

where $\nu_{1}=1-\nu$. The elastic plate contains a circular cut-out $r<a, 0<\theta \leq 2 \pi$ in terms of polar coordinates $(x, y)=r(\cos \theta, \sin \theta)$ and on the boundary of the cut-out we may either apply clamped-edge conditions of

$$
w=w_{r}=0, \quad \text { on } r=a, 0<\theta \leq 2 \pi
$$

or free-edge conditions, expressed by

$$
\left.\begin{array}{rl}
\Delta w-\frac{\nu_{1}}{r^{2}}\left(\partial_{\theta \theta}+r \partial_{r}\right) w & =0 \\
\partial_{r} \Delta w+\frac{\nu_{1}}{r^{2}}\left(\partial_{r}-r^{-1}\right) \partial_{\theta \theta} w & =0
\end{array}\right\}
$$

representing the vanishing of bending moment and shear stress.

Finally, we need to specify radiation conditions as $|x| \rightarrow \infty$. Since we are seeking trapped modes, in which the vibrations are localised in the vicinity of the circular cutout, we impose the condition

$$
w, \Delta w \rightarrow 0, \quad \text { as }|x| \rightarrow \infty,|y|<d .
$$


The field equation can be decoupled into two second-order equations by writing

$$
-\Delta v=k^{2} w, \quad-\Delta w=k^{2} v
$$

and then defining $\phi=w+v=w-k^{-2} \Delta w$ and $\psi=w-v=v+k^{-2} \Delta w$, so that $w=\frac{1}{2}(\phi+\psi)$ and $v=\frac{1}{2}(\phi-\psi)$ we have

$$
\text { (a) }\left(\Delta+k^{2}\right) \phi=0, \quad \text { and } \quad \text { (b) }\left(\Delta-k^{2}\right) \psi=0
$$

which are uncoupled equations for $\phi$ and $\psi$, satisfying the Helmholtz and modified Helmholtz equations respectively. In general, boundary conditions provide a coupling between $\phi$ and $\psi$, but in the case of simply-supported conditions on $|y|=d$, where $w=\Delta w=0$ (or $v=0)$, the conditions remain uncoupled, via

$$
\phi=0, \quad \psi=0, \quad \text { on }|y|=d,-\infty<x<\infty .
$$

Thus, we may seek independent functions $\phi$ and $\psi$ satisfying (8) and (9); this is crucial to our approach. These functions will ultimately be combined to satisfy the coupling boundary conditions (5) or (6) on $r=a$. The simplicity of the method outlined below is lost if the simply-supported conditions on $|y|=d$ were replaced by, say, clamped edge conditions $\left(w=0, w_{y}=0\right)$ on $|y|=d$.

Due to the symmetry in the geometry, we may consider either solutions satisfying the conditions

$$
w=0, \quad \text { and } \quad w_{y y}+\nu w_{x x} \equiv w_{y y}=0, \quad \text { on } y=0
$$

or

$$
w_{y}=0, \quad \text { and } \quad w_{y y y}+(2-\nu) w_{y x x} \equiv w_{y y y}=0, \quad \text { on } y=0
$$

hereafter referred to as antisymmetric and symmetric modes (respectively). In terms of the uncoupled functions $\phi$ and $\psi$ these are

$$
\text { (a) } \phi=\psi=0, \quad \text { or } \quad \text { (b) } \phi_{y}=\psi_{y}=0, \quad \text { on } y=0 .
$$

The conditions in (10) represents physically the fact that the displacement and bending moment vanish along the centreline of the waveguide, whilst in (11) it is the gradient and the shear which vanish.

It proves useful to consider separation solutions for the uniform strip in the absence of any circular cut-out. For example, the solutions in the regions $a<|x|<\infty$ exterior to the cut-out could by represented by superposition of separation solutions, although we do not employ this strategy in our solution.

In the first (antisymmetric) case, separation solutions for $\phi$ and $\psi$ are given, respectively, by

$$
\left\{\sin \left(p_{n} y\right) \mathrm{e}^{ \pm \mathrm{i} k_{n} x}\right\} \quad \text { and } \quad\left\{\sin \left(p_{n} y\right) \mathrm{e}^{ \pm \kappa_{n} x}\right\}, \quad \text { for } n \geq 1
$$

where $p_{n}=n \pi / d$ and $k_{n}=\left(k^{2}-p_{n}^{2}\right)^{1 / 2}=-\mathrm{i}\left(p_{n}^{2}-k^{2}\right)^{1 / 2}$ whilst $\kappa_{n}=\left(k^{2}+p_{n}^{2}\right)^{1 / 2}$. Thus, propagating modes only exist provided $k d<\pi$. The frequency $\omega_{c}$ defined by the relationship $k d=\pi$ (i.e. $\omega_{c}^{2}=D \pi^{4} / m d^{4}$ ) is called the cut-off frequency, and thus $k d<\pi$ implies $\omega<\omega_{c}$, or that the frequency is below the cut-off frequency. In the second (symmetric) case, separation solutions for $\phi$ and $\psi$ are given, respectively, by

$$
\left\{\cos \left(p_{n} y\right) \mathrm{e}^{ \pm \mathrm{i} k_{n} x}\right\} \quad \text { and } \quad\left\{\cos \left(p_{n} y\right) \mathrm{e}^{ \pm \kappa_{n} x}\right\}, \quad \text { for } n \geq 0
$$

where we have reused the variables $p_{n}=\left(n+\frac{1}{2}\right) \pi / d$ and $k_{n}=\left(k^{2}-p_{n}^{2}\right)^{1 / 2}=-\mathrm{i}\left(p_{n}^{2}-k^{2}\right)^{1 / 2}$ whilst $\kappa_{n}=\left(k^{2}+p_{n}^{2}\right)^{1 / 2}$. Now the cut-off frequency $\omega_{c}$ is defined by the relationship 
$k d=\frac{1}{2} \pi$ and for frequencies below the cut-off (i.e. for $k d<\frac{1}{2} \pi$ ), no bounded waves are able to propagate to infinity along the strip.

We briefly remark that in the case of a strip with clamped edges along $|y|=d$, the separation solutions are much more complicated, although cut-off frequencies do exist for both modes of symmetry about $y=0$.

The existence of cut-off frequencies below which no propagating waves exist for a uniform waveguide implies the possibility of trapping modes for frequencies $\omega<\omega_{c}$ represented by local vibrations in the presence of an inhomogeneity within the strip. This is being provided here by a circular cut-out. We already have four different permutations to consider due to the two choices of boundary condition on $r=a$ combined with the choice of symmetry/antisymmetry condition on $y=0$. A further set of combinations may also be introduced by exploiting the symmetry of the geometry about $x=0$. Thus, we may either choose to impose $\phi=\psi=0$ or $\phi_{y}=\psi_{y}=0$ on $x=0$. Previous work on trapping modes suggests that the latter condition is more likely to result in the existence of a trapping mode, and we concentrate on this case in what follows.

\section{A solution using the multipole technique}

In this section we consider just one particular symmetry configuration in detail and in the next section, all possible remaining symmetry combinations are briefly outlined.

In accordance with the earlier decomposition of $w=\phi+\psi$, we may write the total displacement, satisfying (1), (4), using superposition as

$$
w(x, y)=\sum_{n=0}^{\infty} a_{n} \frac{\phi_{2 n+1}^{a}(r, \theta)}{Y_{2 n+1}^{\prime}(\mu)}+b_{n} \frac{\psi_{2 n+1}^{a}(r, \theta)}{K_{2 n+1}^{\prime}(\mu)}, \quad \mu=k a
$$

where $\phi_{2 n+1}^{a}$ and $\psi_{2 n+1}^{a}$ are so-called multipole potentials defined to satisfy, respectively (8a) and (8b) in addition to the boundary conditions (9). The superscript $a$ refers to the fact that the antisymmetry condition (12a) is being applied. The expansion coefficients $a_{n}, b_{n}$ will ultimately be determined by imposing boundary conditions on $r=a$, which is the only point at which the separate parts of the solution become coupled. The normalising factors involve derivatives of the modified Bessel function $K_{2 n+1}$ and the second-kind Bessel function $Y_{2 n+1}$ whose argument is $\mu=k a$.

The multipole potentials are constructed to be modifications to include the conditions (9) to the singular solutions of (8) which apply the absence of any boundaries. These are

$$
H_{n}(k r)\{\cos n \theta, \sin n \theta\}, \quad \text { and } \quad K_{n}(k r)\{\cos n \theta, \sin n \theta\}, \quad n \in \mathbb{N}
$$

where $H_{n}(z)=J_{n}(z)+\mathrm{i} Y_{n}(z)$ is the first-kind Hankel function and $J_{n}(z)$ is the Bessel function. In the case of modes antisymmetric about $y=0(\theta=0, \pi)$ and symmetric about $x=0\left(\theta= \pm \frac{1}{2} \pi\right)$, we select the functions $H_{2 n+1}(k r) \sin (2 n+1) \theta$ and $K_{2 n+1}(k r) \sin (2 n+$ 1) $\theta$. Because of the symmetry, we need consider functions and modifications to them in $0<\theta<\frac{1}{2} \pi$.

The multipole method involves using integral representations of the singular solutions to express them in terms of Cartesian coordinates which then allows them to be modified to include the lateral boundary conditions. Thus, we use (see, for example, [20] , p.20)

$$
H_{n}(z)=\frac{1}{\pi} \int_{\alpha+\mathrm{i} \infty}^{\beta-\mathrm{i} \infty} \mathrm{e}^{\mathrm{i} z \cos s} \mathrm{e}^{\mathrm{i} n(\alpha-\pi / 2)} \mathrm{d} s
$$


for $-\pi<\alpha<0,0<\beta<\pi$ and make the change of variable $s=-\mathrm{i}(v-\mathrm{i} \theta)$ for $0<\theta<\pi$. Then it can be shown, following Appendix A of [19] (but noting a series of obvious typographical errors in that paper), that

$$
H_{2 n+1}(k r) \sin (2 n+1) \theta=-\frac{\mathrm{i}(-1)^{n}}{\pi} \int_{-\infty}^{\infty+\mathrm{i} \pi} \mathrm{e}^{\gamma y} \mathrm{e}^{-(2 n+1) v} \cos (k x \cosh v) \mathrm{d} v
$$

where $\gamma=k \sinh v$. This representation is only valid for $y>0$ or $0<\theta<\pi$; antisymmetry about $y=0$ provides the extension into $y<0$ (alternatively $\mathrm{e}^{\gamma y}$ could be replaced by $\operatorname{sgn}(y) \mathrm{e}^{\gamma|y|}$.) Noting the form of the above expression, we define the multipole by adding to (14), a function satisfying (8a), and the required conditions on $y=0$ and $x=0$, such that (9) is now also satisfied,

$$
\begin{aligned}
\phi_{2 n+1}^{a}(r, \theta)=-\mathrm{i} H_{2 n+1}(k r) \sin (2 n+1) \theta & \\
& +\frac{(-1)^{n}}{\pi} \int_{-\infty}^{\infty+\mathrm{i} \pi} \frac{\mathrm{e}^{\gamma d} \sinh \gamma y}{\sinh \gamma d} \mathrm{e}^{-(2 n+1) v} \cos (k x \cosh v) \mathrm{d} v
\end{aligned}
$$

(the multiplicative factor of $-\mathrm{i}$ is included for later convenience). We note that the denominator in the integrand is zero if $\sinh (k d \sinh v)=0$, which can only happen on the segment $v=i u, 0<u<\pi$ of the integration contour. That is, when $k d \sin u=n \pi$, $n \in \mathbb{Z}$ so and for frequencies below the cut-off when $k d<\pi$, there are no poles in the denominator of the integrand and the integral is well-defined.

The final part of the procedure is to re-expand the integrand in (15) into polar coordinates. Starting with the generating function for the Bessel function ([21], §9.1.41)

$$
\mathrm{e}^{\frac{1}{2} z(t-1 / t)}=J_{0}(z)+\sum_{m=1}^{\infty}\left(t^{m}+(-1)^{m} t^{-m}\right) J_{m}(z)
$$

and substituting $t=\mathrm{ie}^{\mathrm{i}(\theta \pm i v)}, z=k r$ we find, using $\frac{1}{2}(t-1 / t)=\mathrm{i} \cos (\theta \pm \mathrm{i} v)=$ $\mathrm{i} \cos \theta \cosh v \pm \sin \theta \sinh v$. Then, after some algebra,

$$
\begin{aligned}
\mathrm{e}^{\mathrm{i} k x \cosh v} \sinh \gamma y & =\frac{1}{2} \mathrm{e}^{\mathrm{i} k r \cos (\theta+\mathrm{i} v)}-\frac{1}{2} \mathrm{e}^{\mathrm{i} k r \cos (\theta-\mathrm{i} v)} \\
& =-2 \mathrm{i} \sum_{m=1}^{\infty} \mathrm{i}^{m} \sinh m v \sin m \theta J_{m}(k r)
\end{aligned}
$$

(whilst $x \rightarrow-x$ corresponds to $\theta \rightarrow \pi-\theta$ ). The integrand in (15) can therefore be expressed in terms of $(r, \theta)$ only and we have

$$
\phi_{2 n+1}^{a}(r, \theta)=-\mathrm{i} H_{2 n+1}(k r) \sin (2 n+1) \theta+\sum_{m=0}^{\infty} \widehat{A}_{2 m+1,2 n+1} J_{2 m+1}(k r) \sin (2 m+1) \theta
$$

(valid for $r<2 d$; see, for example, [22]) where

$$
\widehat{A}_{2 m+1,2 n+1}=\frac{2(-1)^{m+n}}{\pi} \int_{-\infty}^{\infty+\pi \mathrm{i}} \frac{\mathrm{e}^{\gamma d}}{\sinh \gamma d} \mathrm{e}^{-(2 n+1) v} \sinh (2 m+1) v \mathrm{~d} v .
$$

Dividing the contour of integration into three segments $(-\infty, 0) \cup(0, \pi \mathrm{i}) \cup(\pi \mathrm{i}, \pi \mathrm{i}+\infty)$ and rearranging we find, after some algebra, $\widehat{A}_{2 m+1,2 n+1}=\mathrm{i} \delta_{m n}+A_{2 m+1,2 n+1}$ where $\delta_{m n}$ is the Kronecker delta symbol and 


$$
\begin{aligned}
A_{2 m+1,2 n+1}=\frac{4(-1)^{m+n}}{\pi} \int_{0}^{\infty} \frac{\mathrm{e}^{-\gamma d}}{\sinh \gamma d} \sinh (2 n+1) v \sinh (2 m+1) v \mathrm{~d} v \\
+\frac{4}{\pi} \int_{0}^{\frac{1}{2} \pi} \cot (k d \cos u) \cos (2 n+1) u \cos (2 m+1) u \mathrm{~d} u .
\end{aligned}
$$

Using this in (17) gives

$$
\phi_{2 n+1}^{a}(r, \theta)=Y_{2 n+1}(k r) \sin (2 n+1) \theta+\sum_{m=1}^{\infty} A_{2 m+1,2 n+1} J_{2 m+1}(k r) \sin (2 m+1) \theta
$$

which is real, provided $k d<\pi$.

Next, we have to carry out the same procedure to derive multipoles $\psi_{n}^{a}$ corresponding to singular solutions $K_{2 n+1}(k r) \sin (2 n+1) \theta$. Thus, we start with the integral representation,

$$
K_{n}(z)=\frac{1}{2} \mathrm{i} \int_{\alpha+\mathrm{i} \infty}^{\beta-\mathrm{i} \infty} \mathrm{e}^{-z \cos s} \mathrm{e}^{\mathrm{i} n s} \mathrm{~d} s
$$

([20], p.24) for $-\frac{1}{2} \pi<\alpha, \beta<\frac{1}{2} \pi$ and make the substitution $z=k r, s=-\mathrm{i} v-\theta+\frac{1}{2} \pi$ for $0<\theta<\pi$ with $\alpha, \beta$ chosen such that $\alpha+\theta=0, \beta+\theta=0$. This results in

$$
K_{n}(k r) \mathrm{e}^{\mathrm{i} n \theta}=\frac{1}{2} \mathrm{i}^{n} \int_{-\infty}^{\infty} \mathrm{e}^{-\lambda y} \mathrm{e}^{-\mathrm{i} k x \sinh v} \mathrm{e}^{n v} \mathrm{~d} v
$$

where $\lambda=k \cosh v$. It then follows that

$$
K_{2 n+1}(k r) \sin (2 n+1) \theta=(-1)^{n} \int_{0}^{\infty} \mathrm{e}^{-\lambda y} \cos (k x \sinh v) \cosh (2 n+1) v \mathrm{~d} v .
$$

In this case the multipoles are defined to be

$$
\begin{aligned}
\psi_{2 n+1}^{a}(r, \theta)=K_{2 n+1}(k r) & \sin (2 n+1) \theta \\
& -(-1)^{n} \int_{0}^{\infty} \frac{\mathrm{e}^{-\lambda d} \sinh \lambda y}{\sinh \lambda d} \cos (k x \sinh v) \cosh (2 n+1) v \mathrm{~d} v
\end{aligned}
$$

in which the additional integral satisfies the modified Helmholtz equation, antisymmetry on $y=0$ and symmetry on $x=0$. The function $\psi_{2 n+1}^{a}$ now also satisfies the condition $\psi_{2 n+1}^{a}=0$ on $|y|=d$.

As before we aim to re-expand the integrand of (21) in terms of polar coordinates. Starting with the generating function for the modified Bessel function ([21], §9.6.33)

$$
\mathrm{e}^{\frac{1}{2} z(t+1 / t)}=I_{0}(z)+\sum_{m=1}^{\infty}\left(t^{m}+t^{-m}\right) I_{m}(z)
$$

and substituting $t=\mathrm{ie}^{(v \pm \mathrm{i} \theta)}, z=k r$ we find, $\frac{1}{2}(t+1 / t)=\mathrm{i} \sinh (v \pm \mathrm{i} \theta)=\mathrm{i} \sinh v \cos \theta \mp$ $\cosh v \sin \theta$ and then

$$
\begin{aligned}
\mathrm{e}^{\mathrm{i} k x \sinh v} \sinh \lambda y= & \frac{1}{2} \mathrm{e}^{\mathrm{i} k r \sinh (v-\mathrm{i} \theta)}-\frac{1}{2} \mathrm{e}^{\mathrm{i} k r \sinh (v+\mathrm{i} \theta)} \\
= & -2 \mathrm{i} \sum_{m=1}^{\infty}(-1)^{m} \sinh 2 m v \sin 2 m \theta I_{2 m}(k r) \\
& +2 \sum_{m=0}^{\infty}(-1)^{m} \cosh (2 m+1) v \sin (2 m+1) \theta I_{2 m+1}(k r)
\end{aligned}
$$


from which the integrand in (21) can be expressed in terms of $(r, \theta)$ only and we have

$$
\psi_{2 n+1}^{a}(r, \theta)=K_{2 n+1}(k r) \sin (2 n+1) \theta+\sum_{m=0}^{\infty} B_{2 m+1,2 n+1} I_{2 m+1}(k r) \sin (2 m+1) \theta
$$

where

$$
B_{2 m+1,2 n+1}=-2(-1)^{m+n} \int_{0}^{\infty} \frac{\mathrm{e}^{-\lambda d}}{\sinh \lambda d} \cosh (2 m+1) v \cosh (2 n+1) v \mathrm{~d} v .
$$

The general solution in (13) satisfying (1) and (4) can now be written entirely in terms of $(r, \theta)$ using (19) and (23). We are therefore in a position to apply the final boundary conditions of the problem which apply on $r=a$. In the simpler case of clamped edge conditions, application of (5) and using the orthogonality of $\sin (2 m+1) \theta$ over $0<\theta<\frac{1}{2} \pi$ gives

$$
\begin{aligned}
a_{m} \frac{Y_{2 m+1}(\mu)}{Y_{2 m+1}^{\prime}(\mu)}+b_{m} & \frac{K_{2 m+1}(\mu)}{K_{2 m+1}^{\prime}(\mu)} \\
& +\sum_{n=0}^{\infty} a_{n} \frac{J_{2 m+1}(\mu)}{Y_{2 n+1}^{\prime}(\mu)} A_{2 m+1,2 n+1}+\sum_{n=0}^{\infty} b_{n} \frac{I_{2 m+1}(\mu)}{K_{2 n+1}^{\prime}(\mu)} B_{2 m+1,2 n+1}=0
\end{aligned}
$$

and

$$
a_{m}+b_{m}+\sum_{n=0}^{\infty} a_{n} \frac{J_{2 m+1}^{\prime}(\mu)}{Y_{2 n+1}^{\prime}(\mu)} A_{2 m+1,2 n+1}+\sum_{n=0}^{\infty} b_{n} \frac{I_{2 m+1}^{\prime}(\mu)}{K_{2 n+1}^{\prime}(\mu)} B_{2 m+1,2 n+1}=0 .
$$

Trapped modes correspond to non-trivial solutions of the real infinite homogeneous system of coupled equations above, for $k d<\pi$. It proves useful to express these equations in a canonical form

$$
\sum_{n=0}^{\infty} a_{n} C_{2 m+1,2 n+1}+\sum_{n=0}^{\infty} b_{n} D_{2 m+1,2 n+1}=0
$$

and

$$
\sum_{n=0}^{\infty} a_{n} E_{2 m+1,2 n+1}+\sum_{n=0}^{\infty} b_{n} F_{2 m+1,2 n+1}=0
$$

in which

$$
\begin{aligned}
C_{m, n}=\frac{Y_{m}(\mu)}{Y_{m}^{\prime}(\mu)} \delta_{m n}+\frac{J_{m}(\mu)}{Y_{n}^{\prime}(\mu)} A_{m, n}, & E_{m, n}=\delta_{m n}+\frac{J_{m}^{\prime}(\mu)}{Y_{n}^{\prime}(\mu)} A_{m, n}, \\
D_{m, n}=\frac{K_{m}(\mu)}{K_{m}^{\prime}(\mu)} \delta_{m n}+\frac{I_{m}(\mu)}{K_{n}^{\prime}(\mu)} B_{m, n}, & F_{m, n}=\delta_{m n}+\frac{I_{m}^{\prime}(\mu)}{K_{n}^{\prime}(\mu)} B_{m, n} .
\end{aligned}
$$

In the case of free edges, where we apply (6) to (13) with (19) and (23), the situation is algebraically more complicated. We are aided by the fact that, by definition, $\Delta \phi_{n}^{a}=$ $-k^{2} \phi_{n}^{a}$ applied. Similarly, $\Delta \psi_{n}^{a}=k^{2} \psi_{n}^{a}$. Thus, we end up with the same canonical set of equations as above, but with (27) replaced by 


$$
\begin{aligned}
C_{m, n}= & {\left[\left(-\mu^{2}+\nu_{1} m^{2}\right) \frac{Y_{m}(\mu)}{Y_{m}^{\prime}(\mu)}-\nu_{1} \mu\right] \delta_{m n} } \\
+ & {\left[\left(-\mu^{2}+\nu_{1} m^{2}\right) \frac{J_{m}(\mu)}{Y_{n}^{\prime}(\mu)}-\nu_{1} \mu \frac{J_{m}^{\prime}(\mu)}{Y_{n}^{\prime}(\mu)}\right] A_{m, n}, } \\
D_{m, n}= & {\left[\left(\mu^{2}+\nu_{1} m^{2}\right) \frac{K_{m}(\mu)}{K_{m}^{\prime}(\mu)}-\nu_{1} \mu\right] \delta_{m n} } \\
+ & {\left[\left(\mu^{2}+\nu_{1} m^{2}\right) \frac{I_{m}(\mu)}{K_{n}^{\prime}(\mu)}-\nu_{1} \mu \frac{I_{m}^{\prime}(\mu)}{K_{n}^{\prime}(\mu)}\right] B_{m, n}, }
\end{aligned}
$$

and

$$
\begin{aligned}
E_{m, n}= & {\left[-\mu\left(\mu^{2}+\nu_{1} m^{2}\right)+\nu_{1} m^{2} \frac{Y_{m}(\mu)}{Y_{m}^{\prime}(\mu)}\right] \delta_{m n} } \\
+ & {\left[-\mu\left(\mu^{2}+\nu_{1} m^{2}\right) \frac{J_{m}^{\prime}(\mu)}{Y_{n}^{\prime}(\mu)}+\nu_{1} m^{2} \frac{J_{m}(\mu)}{Y_{n}^{\prime}(\mu)}\right] A_{m, n} } \\
F_{m, n}= & {\left[\mu\left(\mu^{2}-\nu_{1} m^{2}\right)+\nu_{1} m^{2} \frac{K_{m}(\mu)}{K_{m}^{\prime}(\mu)}\right] \delta_{m n} } \\
+ & {\left[\mu\left(\mu^{2}-\nu_{1} m^{2}\right) \frac{I_{m}^{\prime}(\mu)}{K_{n}^{\prime}(\mu)}+\nu_{1} m^{2} \frac{I_{m}(\mu)}{K_{n}^{\prime}(\mu)}\right] B_{m, n} . }
\end{aligned}
$$

It should be noted that $A_{m, n}$ and $B_{m, n}$ only depend upon $k d$ and not $\mu=k a$. This is particularly useful for improving the efficiency of the numerical scheme, where most of the effort goes into computing $A_{m, n}$ and $B_{m, n}$, which is independent of $a / d$.

\section{Further solutions for alternative symmetries}

In this section, we briefly outline the method of solution, based on that in the previous section, for all remaining possible modes of symmetry.

For modes which are both antisymmetric about $y=0$ and $x=0$, the multipoles are based upon the singular solutions $H_{2 n}(k r) \sin 2 n \theta, K_{2 n}(k r) \sin 2 n \theta$. In this case the multipoles are given by

$$
\phi_{2 n}^{a}(r, \theta)=-\mathrm{i} H_{2 n}(k r) \sin 2 n \theta-\frac{(-1)^{n}}{\pi} \int_{-\infty}^{\infty+\mathrm{i} \pi} \frac{\mathrm{e}^{\gamma d} \sinh \gamma y}{\sinh \gamma d} \mathrm{e}^{-2 n v} \sin (k x \cosh v) \mathrm{d} v
$$

and

$$
\psi_{2 n}^{a}(r, \theta)=K_{2 n}(k r) \sin 2 n \theta+(-1)^{n} \int_{0}^{\infty} \frac{\mathrm{e}^{-\lambda d} \sinh \gamma y}{\sinh \lambda d} \sin (k x \sinh v) \sinh 2 n v \mathrm{~d} v .
$$

Then, converting the integrands in (30) into polar coordinates, using (16) and (22) we obtain

and

$$
\phi_{2 n}^{a}(r, \theta)=Y_{2 n}(k r) \sin 2 n \theta+\sum_{m=1}^{\infty} A_{2 m, 2 n} J_{2 m}(k r) \sin 2 m \theta
$$

$$
\psi_{2 n}^{a}(r, \theta)=K_{2 n}(k r) \sin 2 n \theta+\sum_{m=1}^{\infty} B_{2 m, 2 n} I_{2 m}(k r) \sin 2 m \theta
$$


where

$$
\begin{aligned}
A_{2 m, 2 n}=\frac{4(-1)^{m+n}}{\pi} \int_{0}^{\infty} \frac{\mathrm{e}^{-\gamma d}}{\sinh \gamma d} \sinh 2 n v & \sinh 2 m v \mathrm{~d} v \\
& +\frac{4}{\pi} \int_{0}^{\frac{1}{2} \pi} \cot (k d \cos u) \sin 2 n u \sin 2 m u \mathrm{~d} u .
\end{aligned}
$$

and

$$
B_{2 m, 2 n}=-2(-1)^{m+n} \int_{0}^{\infty} \frac{\mathrm{e}^{-\gamma d}}{\sinh \gamma d} \sinh 2 n v \sinh 2 m v \mathrm{~d} v .
$$

As before the cut-off frequency is defined by $k d=\pi$ and for $\omega<\omega_{c}$, the multipoles above are real. The general solution, in terms of the multipoles given above and reusing the coefficients $a_{n}$ and $b_{n}$, is written

$$
w(x, y)=\sum_{n=1}^{\infty} a_{n} \frac{\phi_{2 n}^{a}(r, \theta)}{Y_{2 n}^{\prime}(\mu)}+b_{n} \frac{\psi_{2 n}^{a}(r, \theta)}{K_{2 n}^{\prime}(\mu)} .
$$

Application of the clamped edge conditions on $r=a$ gives

$$
\sum_{n=0}^{\infty} a_{n} C_{2 m, 2 n}+\sum_{n=0}^{\infty} b_{n} D_{2 m, 2 n}=0
$$

and

$$
\sum_{n=0}^{\infty} a_{n} E_{2 m, 2 n}+\sum_{n=0}^{\infty} b_{n} F_{2 m, 2 n}=0
$$

using the definitions in (27), whilst the same system holds for the free edge conditions on $r=a$, with matrix elements given instead by (28) and (29).

If symmetric modes are considered, so that (12a) is replaced by $(12 b)$ then a further set of combinations is generated. We start with

$$
\phi_{2 n}^{s}(r, \theta)=-\mathrm{i} H_{2 n}(k r) \cos 2 n \theta+\frac{(-1)^{n}}{\pi} \int_{-\infty}^{\infty+\pi i} \frac{\mathrm{e}^{\gamma d}}{\cosh \gamma d} \cosh \gamma y \cos (k x \sinh v) \mathrm{e}^{-2 n v} \mathrm{~d} v
$$

the additional integral ensuring the symmetry condition holds and such that the condition on $y=d$ is met. Using

$$
\mathrm{e}^{\mathrm{i} k x \sinh v} \cosh \gamma y=J_{0}(k r)+2 \sum_{m=1}^{\infty} \mathrm{i}^{m} \cosh m v \cos m \theta J_{m}(k r)
$$

gives

$$
\phi_{2 n}^{s}(r, \theta)=Y_{2 n}(k r) \cos 2 n \theta+\sum_{m=0}^{\infty} A_{2 m, 2 n} J_{2 m}(k r) \cos 2 m \theta
$$

where

$$
\begin{aligned}
A_{2 m, 2 n}=\frac{2 \epsilon_{m}(-1)^{m+n}}{\pi} \int_{0}^{\infty} \frac{\mathrm{e}^{-\gamma d}}{\cosh \gamma d} \cosh 2 n v \cosh 2 m v \mathrm{~d} v \\
\quad-\frac{2 \epsilon_{m}}{\pi} \int_{0}^{\frac{1}{2} \pi} \tan (k d \cos u) \cos 2 m u \cos 2 n u \mathrm{~d} u
\end{aligned}
$$


is real provided $k d<\frac{1}{2} \pi$. In the above $\epsilon_{0}=1$ and $\epsilon_{n}=2$ for $n \geq 1$. Also,

$$
\psi_{2 n}^{s}(r, \theta)=K_{2 n}(k r) \cos 2 n \theta+\sum_{m=0}^{\infty} B_{2 m, 2 n} I_{2 m}(k r) \cos 2 m \theta
$$

where

$$
B_{2 m, 2 n}=-\epsilon_{m}(-1)^{m+n} \int_{0}^{\infty} \frac{\mathrm{e}^{-\lambda d}}{\cosh \lambda d} \cosh 2 m v \cosh 2 n v \mathrm{~d} v .
$$

Finally, for symmetry about $y=0$ and antisymmetry about $x=0$ we have

$$
\phi_{2 n+1}^{s}(r, \theta)=Y_{2 n+1}(k r) \cos (2 n+1) \theta+\sum_{m=0}^{\infty} A_{2 m+1,2 n+1} J_{2 m+1}(k r) \cos (2 m+1) \theta
$$

where

$$
\begin{aligned}
A_{2 m+1,2 n+1}=\frac{4(-1)^{m+n}}{\pi} \int_{0}^{\infty} & \frac{\mathrm{e}^{-\gamma d}}{\cosh \gamma d} \cosh (2 n+1) v \cosh (2 m+1) v \mathrm{~d} v \\
& \quad-\frac{4}{\pi} \int_{0}^{\frac{1}{2} \pi} \tan (k d \cos u) \sin (2 m+1) u \sin (2 n+1) u \mathrm{~d} u
\end{aligned}
$$

whilst

$$
\psi_{2 n+1}^{s}(r, \theta)=K_{2 n+1}(k r) \cos (2 n+1) \theta+\sum_{m=0}^{\infty} B_{2 m+1,2 n+1} I_{2 m+1}(k r) \cos (2 m+1) \theta
$$

where

$$
B_{2 m+1,2 n+1}=-2(-1)^{m+n} \int_{0}^{\infty} \frac{\mathrm{e}^{-\lambda d}}{\cosh \lambda d} \sinh (2 m+1) v \sinh (2 n+1) v \mathrm{~d} v .
$$

Application of clamped edge or free edge conditions to either of the symmetric modes considered above leads to systems of equations for the unknown expansion coefficients as before.

\section{Results}

Mathematically, trapped waves correspond to the vanishing of the infinite determinant of the real matrix defined by combining the two systems of linear equations in either (25), (26) or (33), (34), depending on the symmetry. In the computation of the trapped waves, each system of equations is truncated to include $N+1$ coefficients $a_{n}$ and $b_{n}$. In other words we seek a zero of the real determinant of a $(2 N+2)$-square matrix. The elements of the matrix require the computation of the coefficients $A_{m, n}$ and $B_{m, n}$, either via (27) for clamped edges or via (28), (29) for free edges on the circle $r=a$ and where $m, n$ are either even or odd, depending upon the symmetry being considered. We note that $A_{m, n}$ and $B_{m, n}$ depend only on $k d$ and have symmetries in $m$ and $n$, whilst the independent parameter $a / d$ is used only in defining the matrix elements. These facts can be used to improve the numerical efficiency. The factors $A_{m, n}$ and $B_{m, n}$ are evaluated numerically using a basic Guassian quadrature scheme; the infinite integrals decay expontentially at infinity.

The value of truncation parameter $N$ used depends upon $a / d$. Numerical experimentation suggests that for $a / d<0.75$, a value of $N=12$ ensures five place accuracy in the 


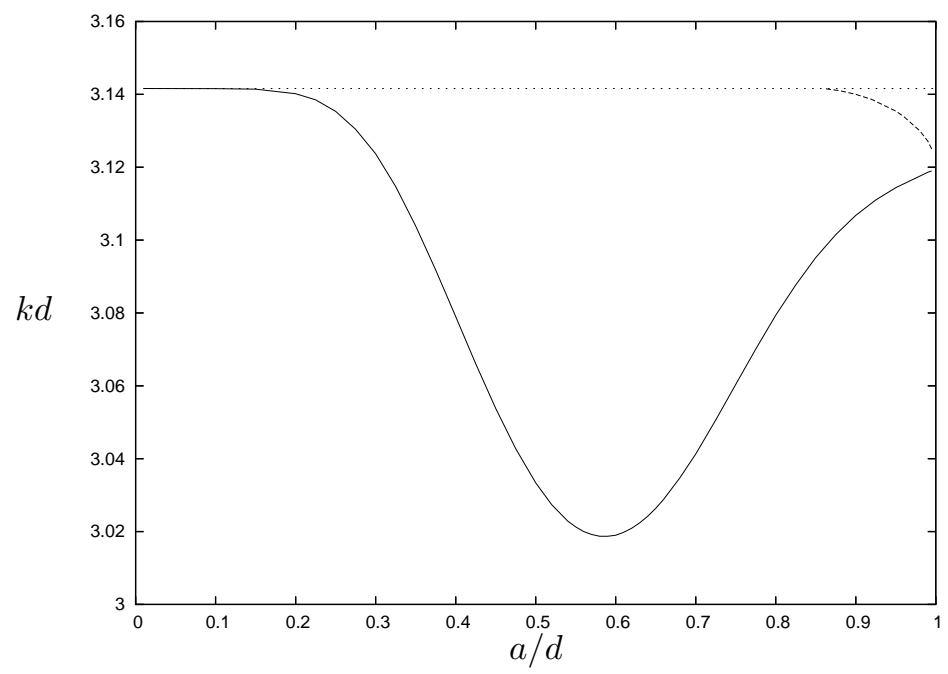

Fig. 2. Relationship between wavenumber, $k d$, and size of circle, $a / d$, for trapped waves with antisymmetry in $y$. Solid/dashed lines refer to symmetry/antisymmetry in $x$

value of the trapped wave frequencies, whilst larger values of $N$ are required for similar accuracy as $a / d \rightarrow 1$.

No evidence of trapped waves were found for a hole which is clamped, for each of the four symmetries. In contrast, when the hole has a free edge, trapped waves have been located (numerically) in each of the four symmetries. The results, shown in terms of the two paramaters $k d$ and $a / d$ are presented in figures 1 and 2. Thus, in the first figure, we consider modes which are antisymmetric about the centre of the guide, and either symmetric (solid line) or antisymmetric (dashed line) about the line $x=0$. Also shown is the non-dimensional cut-off wavenumber $k d=\pi$. The symmetric mode exists for all $a / d$, whilst the antisymmetric mode only exists for $a / d>0.86$. The two curves appear to approach one another as $a / d \rightarrow 1$. In this limit, the guide is divided in two by the circle, and the notion of symmetry and antisymmetry is lost.

Similar qualitative results have been reported for circles in acoustic waveguides (equivalent to circular cylinders in channels in the theory of water waves) by [19] and [23] who computed the modes with symmetry and antisymmetry about the $x=0$ line.

In figure 3, the same set of results are shown for modes which are symmetric about the centre of the guide. Here, the cut-off wavenumber is $k d=\frac{1}{2} \pi$ (dotted line). Again, modes with symmetry in $x$ exist for all $a / d$, whilst those antisymmetric in $x$ can only just be seen in figure 3 , in the top right hand corner, existing for $a / d \geq 0.99$. Again, these two curves appear to meet as $a / d \rightarrow 1$.

An illustration of the modal shape of the trapped wave is given in figure 4 ; see figure caption for parameters. The amplitude of the deflection vanishes as $|x| \rightarrow \infty$.

\section{Conclusions}

In this paper, we have provided numerical evidence for new examples of trapped waves on thin elastic plates. It has been shown that trapped waves exist for circular cut-outs with free edges, of all radii, centrally placed within a waveguide with simply supported 


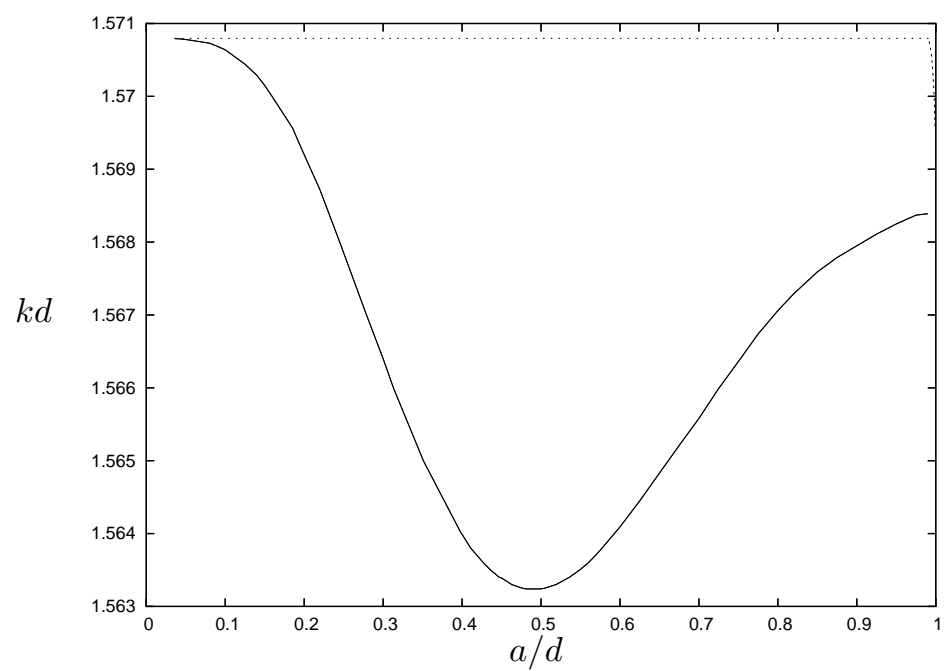

Fig. 3. Relationship between wavenumber, $k d$, and size of circle, $a / d$, for trapped waves with symmetry in $y$. Solid/dashed lines refer to symmetry/antisymmetry in $x$.

conditions placed upon the lateral boundaries. These conditions imply, using image arguments, the existence of trapped waves for an infinite periodic array of circular holes in an infinite thin elastic plate. In this context, the practical importance of trapped waves is that large resonances can be expected to occur for large finite linear periodic arrays of circular holes, in accordance with [24] work showing the relationship between trapped waves in acoustic waveguides and acoustic scattering by large periodic arrays.

There are a range of extensions to the current work that can be considered. The simplysupported conditions on the lateral boundaries of the waveguide could be replaced with more realistic clamped or free edge conditions. It can be shown that, in either case, there is a non-zero cut-off frequency. However, as pointed out in the text, the method of solution would require a non-trivial modification. Secondly, we have shown that the circular cut-out requires free edge conditions to support trapped waves, whilst in the Introduction we describe wave trapping by dividing the guide in two with a free-edged cut perpendicular to the guide. It might therefore be informative to consider the effect of a cut perpendicular to the guide but which only partially extends across the guide, a relatively simple problem to investigate mathematically. Finally, it should be possible to provide rigourous existence and non-existence proofs for trapped waves, possibly using variational methods similar to those used by [25] in proving the existence of trapped waves in acoustic waveguides.

\section{Acknowledgements}

RP would like to thank Prof. David Evans for useful discussions. 


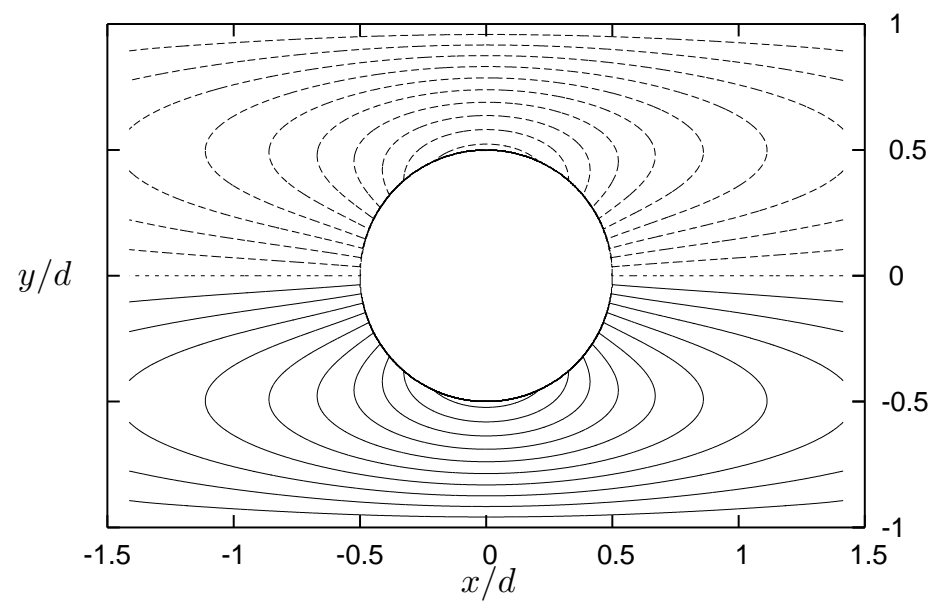

Fig. 4. Contour plot of the trapped wave deflection amplitude $w(x, y)$, antisymmetric about $y=0$ and symmetric about $x=0$. Solid/broken contours refer to positive/negative values of $w$. Trapped wave parameters: $a / d=\frac{1}{2}, k d=3.03336$.

\section{References}

[1] G.A. Kriegsmann, Scattering matrix analysis of a photonic Fabry-Perot resonator, Wave Motion 37 (2003) 43-61.

[2] G.G. Stokes, Report on recent researches in hydrodynamics, Brit. Assn Rep. (1846)

[3] M. McIver, An example of non-uniqueness in the two-dimensional linear water wave problem, J. Fluid Mech. 315 (1996) 257-266.

[4] J.D. Kaplunov, G.A. Rogerson \& P.E. Tovstik, Localized vibration in elastic structures with slowly varying thickness, Quart. J. Mech. Appl. Math. 58 (2005) 645-664.

[5] D. Gridin, R.V. Craster \& A.T.I. Adamou, Trapped modes in curved elastic plates, Proc. Roy. Soc. Lond A. 461 (2005) 1181-1197.

[6] J. Postnova \& R.V. Craster, Trapped modes in topographically varying elastic waveguides, To appear in Wave Motion.

[7] S. Timoshenko \& S. Woinowsky-Krieger, Theory of Plates and Shells (2nd Ed.) (1959) New York: McGraw-Hill.

[8] Yu. K. Konenkov, A Rayleigh-type flexural wave, Sov. Phys. Acoust. 6 (1960) 122-123.

[9] R.N. Thurston \& J. McKenna, Flexural acoustic waves along the edge of a plate, IEEE Trans Sonics Ultranson. 21 (1974) 296-297.

[10] A.N. Norris \& Z. Wang, Bending-wave diffraction from strips and cracks on thin plates, Quart. J. Mech. Appl. Math. 47 (1994) 607-627.

[11] A.N. Norris, Flexural edge waves, J. Sound Vib. 171 (1994) 571-573.

[12] A.N. Norris, V.V. Krylov \& I.D. Abrahams, Flexural edge waves and comments on 'A new bending wave solution for the classical plate equation', J. Acoust. Soc. Am. 107 (2000) 1781-1784.

[13] I. Thompson, I.D. Abrahams \& A.N. Norris, On the existence of flexural waves on thin orhtotropic plates, J. Acoust. Soc. Am. 112 (2002) 1756-1765.

[14] I.D. Abrahams \& A.N. Norris, On the existence of flexural waves on submerged elastic plates, Proc. Roy. Soc. Lond. A. 456 (2000) 1559-1582.

[15] D.V. Evans \& R. Porter, Wave scattering by narrow cracks in ice sheets floating on water of finite depth, J. Fluid Mech. 484 (2003) 143-165.

[16] D.V. Evans \& R. Porter, Penetration of flexural waves through a periodically constrained thin elastic plates in vacuo and floating on water, To appear in J. Eng. Math. (also available on http://oe.mit.edu/flowlab/newmanbook.html)

[17] P. McIver \& M. McIver, Trapped modes in the water-wave problem for a freely-floating structure, J. Fluid Mech. 558 (2006) 53-67. 
[18] A.N. Norris \& C. Vermula, Scattering of flexural waves on thin plates, J. Sound Vib. 181 (1995) $115-125$.

[19] M. Callan, C.M. Linton \& D.V. Evans, Trapped modes in two-dimensional waveguides, J. Fluid Mech., 229 (1991) 51-64.

[20] A. Erdélyi, W. Magnus, F. Oberhettinger \& F.G. Tricomi, Higher Transcendental Functions, vol. 2. New York: McGraw-Hill (1953).

[21] M.A. Abramowitz \& I. Stegun (eds), Handbook of Mathematical Functions. New York: Dover (1965).

[22] C.M. Linton \& P. McIver, Handbook of Mathematical Techniques for Wave/Structure Interactions, CRC Press (2001).

[23] D.V. Evans \& R. Porter, Trapped modes about multiple cylinders in a channel, J. Fluid Mech. 339 (1997) 331-356.

[24] H.D. Maniar \& J.N. Newman, Wave diffraction by a long array of cylinders, J. Fluid Mech. 339 (1997) 309-330.

[25] D.V. Evans, M. Levitin \& D. Vassiliev, Existence theorems for trapped modes, J. Fluid Mech. 261 (1994) 21-31. 